\title{
Remote Vascular Interventional Surgery Robotics: A Review
}

\author{
Yang Zhao, Ziyang Mei, Xiaoxiao Luo, Jingsong Mao, Qingliang Zhao, Gang Liu and Dezhi Wu
}

\begin{abstract}
Interventional doctors are exposed to radiation hazards during the operation and endure high work intensity. Remote vascular interventional surgery robotics is a hot research field that can not only protect the health of interventional doctors, but also improve accuracy and efficiency of surgeries. However, the current vascular interventional robots still have many shortcomings to be improved. This article introduces the mechanical structure characteristics of various fields of vascular interventional therapy surgical robots, discusses the current key features of vascular interventional surgical robotics in force sensing, haptic feedback, and control methods, summarizes current frontiers about autonomous surgery, long geographic distances remote surgery and MRI-compatible structures. Finally, combined with the current research status of vascular interventional surgery robots, this article analyzes the development directions and puts forward a vision for the future vascular interventional surgery robots.
\end{abstract}

Index Terms-Haptic feedback, Medical robotics, Vascular Interventional.

\section{INTRODUCTION}

$\mathrm{I}$ NTERVENTIONAL radiology has developed over the last several decades, becoming one of the three main effective therapies besides Internal Medicine and Surgery [1]. The benefits of interventional radiology to patients are both extensive and beyond dispute, but many of these procedures also can produce patient radiation doses high enough to cause radiation effects and occupational doses to interventional radiologists high enough to cause concern. To limit occupational radiation dose to an acceptable level, radiologists usually use personal protective devices, such as aprons, thyroid shields, eyewear, and gloves [2]. Those devices are heavy and are a burden to radiologists when performing interventional surgery. This situation makes interventional surgeons face two major health risks: fluorescent radiation and musculoskeletal strain [3].

To protect radiologists from potential health problems caused by fluoroscopy radiation and to minimize the patient radiation doses, in recent decades, many institutes have been working to develop robotic systems aimed to precisely steer and

This work is financially supported by the Project of Youth Innovation Fund of Xiamen City (3502Z20206041), the Fundamental Research Funds for the Central Universities (20720190144), the National Natural Science Foundation of China (52075464), Science and Technology Program of Shenzhen City (JCYJ20180306172700388) and the Science and Technology Project of Xiamen Municipal Bureau of Science and Technology(3502Z20194044). (Corresponding author: Dezhi Wu).

Y. Zhao, Z. Mei, L. Luo and D. Wu are with the Department of mechanical $\&$ electrical engineering, Xiamen University, Xiamen 361102, China. (e-mail: position interventional tools for catheter-based interventional surgeries, such as guidewires, microcatheters, balloons, and stents. Those robotic systems are supposed to shorten procedure time and reduce patient exposure to contrast agents and radiation, allowing operators to perform surgery by a remote console behind a radiation shield. The main advantages of using robotic technology are the increased levels of speed, precision, reproducibility, and endurance of these machines compared with human performance. In medicine, robotic technology has been used since the mid-1990s, primarily in surgery and radiation therapy [4].

Vascular interventional surgical robotics has been developing for many years, but this has been complicated by the challenging therapy methods and surgical procedures and various surgical equipment involved. Common vascular interventional surgical robots are designed for angioplasty, vascular embolization, or radiofrequency ablation. The interventional instruments operated by surgical robots are also different in different surgical scenarios [5-8]. Related commercial products in various fields have been certified and put into clinical use. According to clinical reports, the application of vascular interventional robots has significantly reduced the amount of radiation received by interventional doctors and reduces the work intensity of interventional doctors [9]. The high-precision manipulation characteristics of the robot system shorten the operation time and greatly increase the success rate of the operation.

This review will briefly start from vascular interventional surgery, discuss the structural design of interventional surgery robots in combination with existing research institutions and commercial systems. We discuss the key features of vascular interventional surgery robot technology and the frontiers that are currently being developed and combined.

\section{STRUCTURES OF VASCULAR INTERVENTIONAL ROBOTS}

Interventional surgery robot systems can be roughly divided into general vascular interventional robots and electrophysiological interventional therapy robots from the application field. General vascular interventional includes angioplasty and intravascular infusion. Electrophysiological

zhaoy@xmu.edu.cn, umeko@stu.xmu.edu.cn; luoxiaoxiao@stu.xmu.edu.cn; wdz@xmu.edu.cn).

J. Mao is with Department of Radiology, Xiang'an Hospital of Xiamen University, Xiamen, 361102, China. (e-mail: maojingsong163@163.com).

Q. Zhao and G. Liu are with State Key Laboratory of Molecular Vaccinology and Molecular Diagnostics Center for Molecular Imaging and Translational Medicine, School of Public Health, Xiamen University, Xiamen 361102, China. (e-mail: zhaoql@xmu.edu.cn; gangliu.cmitm@xmu.edu.cn). 
interventional therapy includes interventional radiofrequency ablation. The two types of interventional surgery are very different in terms of treatment methods, treatment purposes, interventional instruments, etc. Therefore, the structures of the two types of surgical robots are very different.

\section{A. General Vasculature Interventional Surgeries}

General vasculature interventional surgeries in interventional robotics can be roughly divided into angioplasty and intravascular infusion.

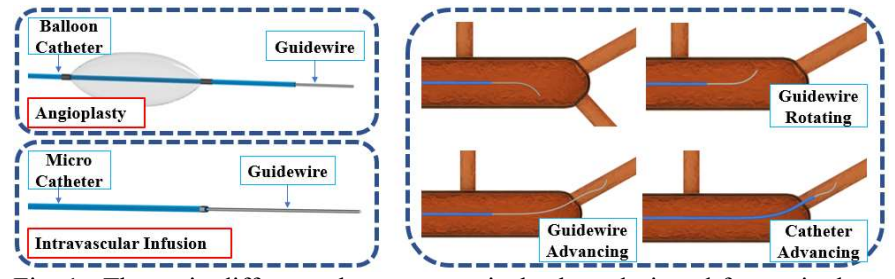

Fig. 1. The main difference between surgical robots designed for angioplasty and intravascular infusion is the interventional instrument being operated. But what they have in common is both of them using a guidewire to guide the catheter to the desired location.

Angioplasty aims to inflate and deploy the balloon after the balloon catheter is sent to the designated location. PCI is a typical application of angioplasty. The goal of intravascular infusion is to deliver the microcatheter to the designated location and then place the drug to the designated location through the microcatheter. TACE is a typical application of intravascular infusion. The main difference between surgical robots designed for angioplasty and intravascular infusion is the manipulation of interventional instruments. Surgical robots designed for angioplasty need to manipulate the balloon catheter and guidewire; surgical robots designed for intravascular infusion need to manipulate the microcatheter and guidewire.

Guo Shuxiang, with his groups at Beijing Institute of Technology and Kagawa University, has designed multiple interventional surgery robot systems. His first-generation system uses a friction wheel mechanism to deliver the interventional device. This generation of robots can only support the two-degree-of-freedom motion of single device [10]. The second-generation system uses a linear slide to deliver the device. It has a wealth of force sensing functions while improving accuracy [11]. The third-generation system uses two sets of linear slides at the same time. While having abundant force-sensing functions, it can also achieve the codelivery of the interventional guidewire and catheter. This generation of the system outperforms human surgeons [12, 13]. Xiao Nan et al. of Beijing Institute of Technology developed the LUBAN interventional surgery robot system based on Guo's thirdgeneration system and completed China's first robot-assisted whole-brain angiography in 2020 [14].

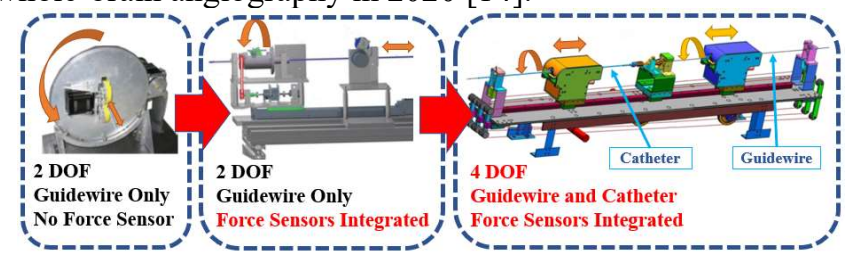

Fig. 2. Three generations designed by Guo et al. With their surgical robot design iterations from generation to generation, the robots integrate richer sensing functions, can control more surgical devices at the same time, and the mechanical design becomes more compact and reliable.
The models and specifications of guidewires and catheters for interventional surgery are very diverse. It is important to be compatible with as many types of interventional devices as possible. Kundong Wang et al. of Shanghai Jiaotong University designed a novel universal endovascular surgical robot. This robotic system was composed of four manipulators with 12 degrees of freedom, which can be compatible with various interventional instruments on the market to complete a variety of different surgical procedures. However, such a mechanism design imposes a stroke limit on the delivery of the catheter or the guidewire. Moreover, the rotation angle of the catheter guidewire is limited, and it is impossible to achieve a full $360^{\circ}$ rotation.

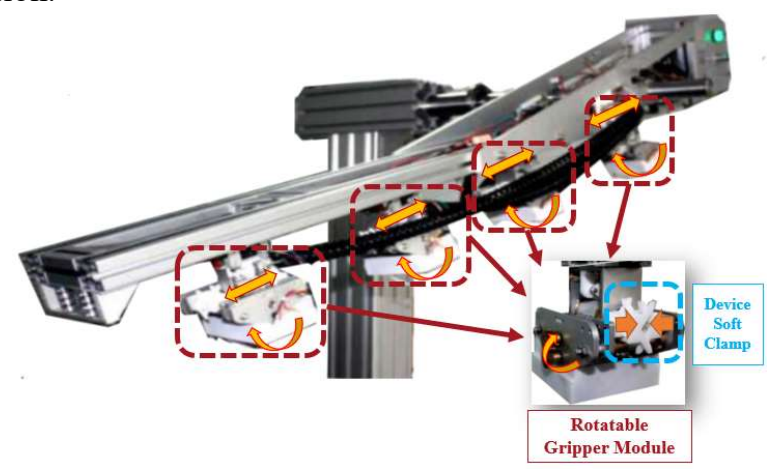

Fig. 2. A universal endovascular robot designed by Kundong Wang et al. Four independent grippers can achieve various action combinations, and the soft clamp can hold interventional instruments of any shape.

Doctors often manipulate the delivery and rotation of the interventional guidewire with one pair of fingers. However, interventional surgical robots often divide delivery and rotation into different modules to complete. Through the design of the friction wheel arrangement, only one pair of friction wheels can simultaneously achieve the rotation and delivery of the guidewire, shown as Fig. 4. This design is very close to the actual doctor's manual operating technique and greatly reduces the volume of the surgical robot. Such a design appeared in a bionic interventional surgery robot designed by Zhenqiu Feng et al. (the Institute of Automation of the Chinese Academy of Sciences) and Hansen's Magellan system.

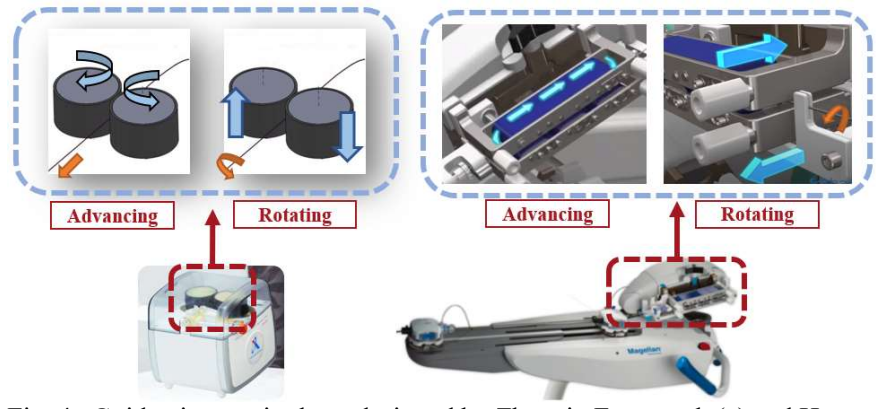

Fig. 4. Guidewire manipulator designed by Zhenqiu Feng et al. (a) and Hansen Medical (b). This design only uses a pair of friction wheels to achieve two kinds of movement of the guidewire, not only there is no stroke restriction on delivery, but the volume is also very small.

Rafael Beyar et al. of Israel's Haifa Center have developed a surgical robot system for PCI based on friction wheel mechanism. This system can operate both the interventional guidewire and the balloon catheter at the same time. The system is very versatile, which has successfully deployed heart stents 
in 18 patients. This surgical robot system is also the predecessor of Corindus' Corpath series surgical robot system [15]. The principle of the current Corpath GRX to drive interventional instruments is still the same as the original. But one very important change is that for hygienic considerations, a large part of the structure is disposable and replaceable. The same goes for Robocath R-One, another commercial interventional surgical robot product [16].

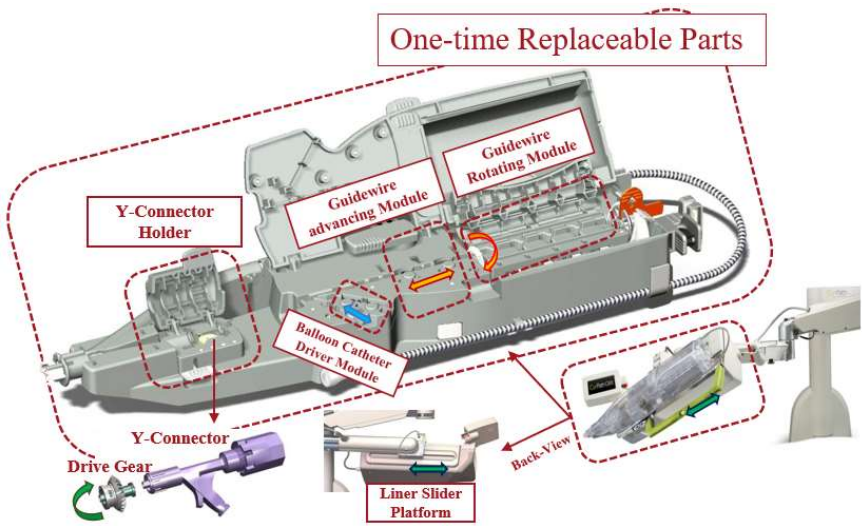

Fig. 5. Commercial robot systems Corpath GRX. It can deliver and rotate the guidewire, deliver the balloon catheter, control the switch of the Y-Connector, and is compatible with PCI and PVI surgery. For hygienic considerations, a large part of the structure is disposable and replaceable.

\section{B. Electrophysiology Therapies}

Interventional electrophysiological therapy has a wide range of applications, and can be used to treat atrial fibrillation, lung tumors, liver tumors and other diseases. Electrophysiological (EP) catheter is the main instrument for electrophysiological surgery. The head tip of the electrophysiological catheter can be flexibly bent. The operation process does not require a guidewire, but under the guidance of steerable electrophysiological catheter itself. The ablation instrument installed on the catheter head tip releases radio frequency current to treat the target tissue $[6,7]$.

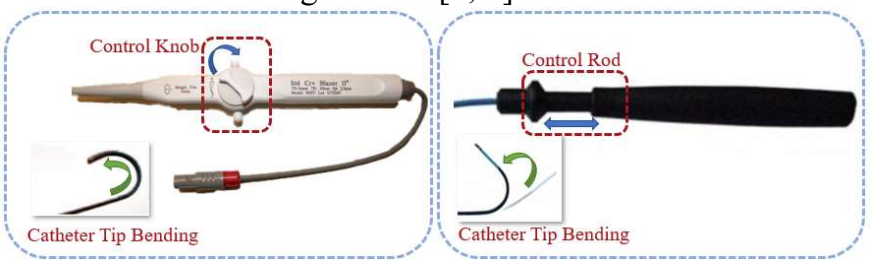

Fig. 6. Electrophysiological (EP) catheters. The handle of the EP catheter has a function that can manipulate its tip to bend.

Interventional surgery robots designed for electrophysiological therapy are mainly to manipulate EP catheters. To be compatible with general electrophysiological catheters, the interventional electrophysiological treatment surgical robot system under development generally adopts a three-degree-of-freedom control design, and the structural design is similar. A common structure provides delivery freedom using a linear moving platform, clamps and rotates the electrophysiological catheter to achieve rotational freedom and designs a special operating mechanism to control the bending freedom of the electrophysiological catheter.

Cercenelli et al. of the University of Bologna designed a highly compact and versatile remote catheter navigation system named CathROB [17-19], which uses two sets of liner slider structures to achieve full control of general electrophysiological catheter. Jun Woo Park et al. of Korea University [20] and Ganji et al. of the University of Waterloo [21] used similar structures to manipulate EP catheters. Their structures include liner slider platform, rotation driver, and steering driver to achieve the translation, rotation, and tip bending motions of EP catheters.

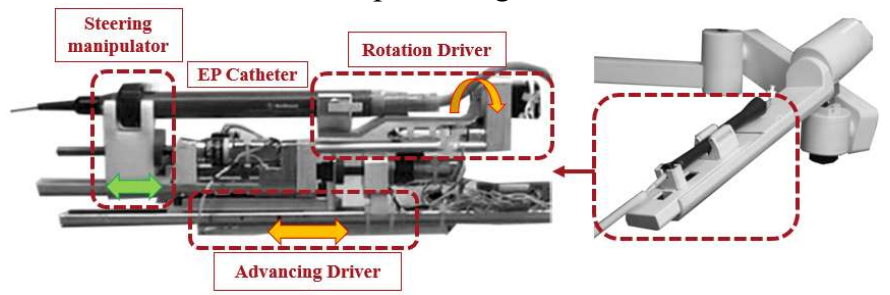

Fig. 7. The mechanical structure of CathROB from Cercenelli et al. The operating handle of an ordinary EP catheter is installed on the robot. Different actuators operate the delivery, rotation, and tip steering of the EP catheter. Many robotic systems have adopted similar schemes to manipulate EP catheters.

In radiofrequency ablation, it is very important for the EP catheter to point to the target tissue accurately and stably. Conventional EP catheter distal bend is driven by ropes. EP catheters driven by magnetic fields can achieve higher flexibility and stability [22]. The Genesis RMN from Stereotaxis uses a specially designed magnetic drive catheter to control the movement of the catheter's front end by changing the external magnetic field [23]. Magnetic navigation has the advantages of high flexibility and strong maneuverability.

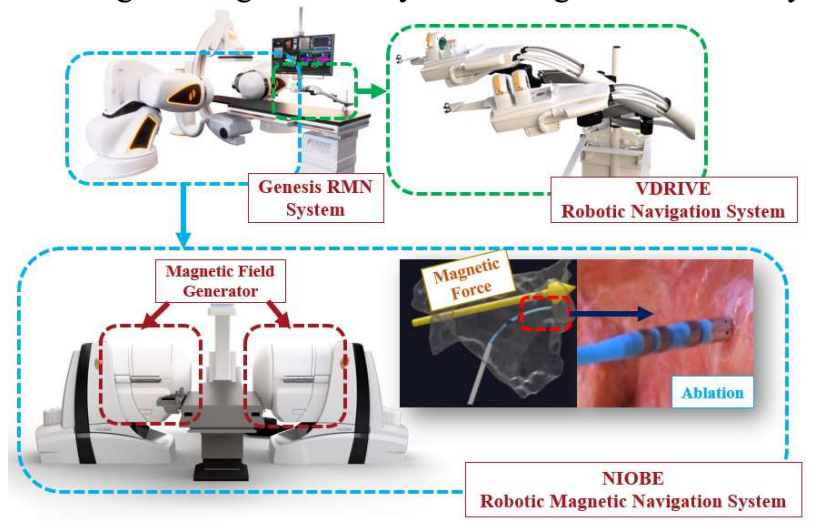

Fig. 8. Stereotaxis' Genesis RMN. Its catheter navigation system named VDrive coordinating with Its Magnetic navigation system named Niobe to complete the operations. By changing the external magnetic field by the magnetic field generator, the catheter tip can be accurately and stably pointed to the target location.

\section{KEY FEATURES OF VASCULAR INTERVENTIONAL ROBOTS}

\section{A. Force Sensing}

The forces that can be collected by interventional surgical instruments are generally divided into two types: the force collected by the tip of the surgical tool, which is in the human body, is called the "distal force", and the force collected by the operating end of the surgical tool outside the human body is called "Proximal force". The distal force is generally composed of the contact force between the surgical tool and the vessel wall, while the proximal force is a complex composite force, including contact force, friction force, and viscous resistance of 
blood.

The measurement of distal force often requires sensors to be placed at the tip of the interventional instrument. This arrangement can directly measure the force between the interventional device and the blood vessel. Sensitive rubbers, strain gauges, and fiber optic pressure sensors are often used to measure distal force. Guo Shuxiang et al. of Kagawa University have proposed various remote sensor arrangement forms in different papers [24-27]. They arrayed pressure-sensitive rubbers into tactile sensors and set them at the distal end of the catheter. Similar schemes are also very common in other research groups, such as Omisore et al. (Shenzhen Institute of Advanced Technology, Chinese Academy of Sciences) [28], Payne et al. (Imperial College of the United Kingdom) [29], and Liu Da et al. (Beijing University of Aeronautics and Astronautics) [30].

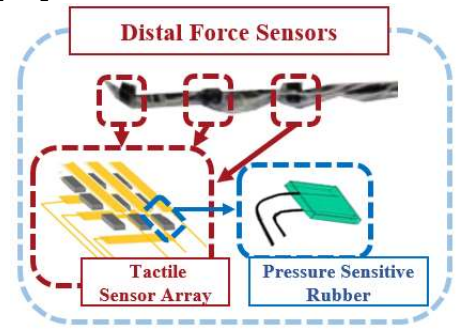

Fig. 9. Distal sensors Sensor arrangement from Guo et al. Array pressuresensitive rubbers into sensing units, arrange them at the distal end of the catheter and encapsulate the lead. Generally, force sensing units are installed at multiple locations on the catheter to monitor the force conditions at different nodes.

In clinical applications, the current distal sensors of interventional instruments are relatively common in electrophysiological (EP) treatments. They are mostly arranged in electrophysiological catheters. Due to the size of the sensor and its lead packaging volume, it is difficult to apply in general vascular interventional operations, especially interventional surgery in small blood vessels. In the field of commercial surgical robots, Hansen Medical's Sensei surgical robot integrates a force sensor on the head of its Artisan active catheter [31].

The force that doctors feel during the operation is mostly the proximal force, and the collection of the proximal force is relatively easy. In the interventional surgical robot, the collection of the proximal force is easier than the distal force. Therefore, the measurement of proximal force appears most frequently in interventional surgery systems. Setting force sensors between the transmission components of the robot is the most widely used approach.

Besides arranging force sensors between the transmission components, Zhou et al. of Xiamen University also tried to arrange a sensing pipe at the front of the robot and indirectly judged the magnitude of the resistance through the squeezing between the interventional device and the sensing pipe [32]. Hyo-Jeong et al. of Hanyang University in South Korea [33] and Sankaran et al. of the University of Illinois [34, 35] measured the resistance forces by the input current of the motors. These methods arrange sensors on the outside of the robot, or even no sensors, and achieve the measurement of resistance force.

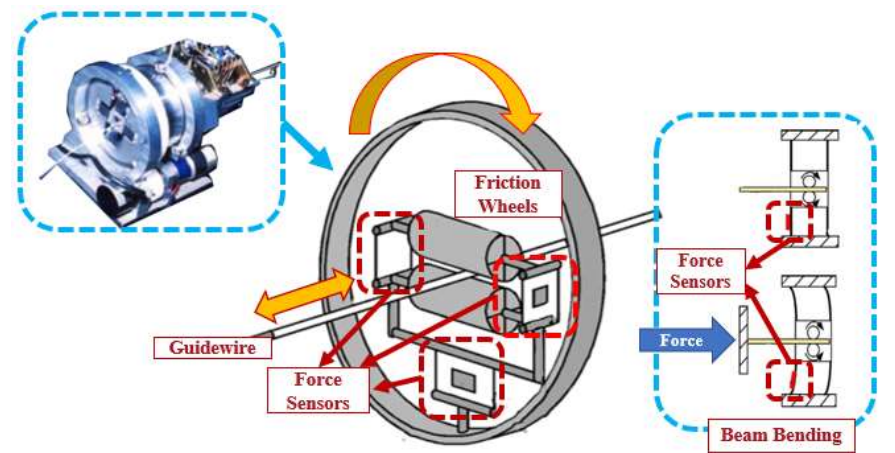

Fig. 10. Proximal sensors arrangement by Arai et al. of Nagoya University [45]. Force sensors are set between the transmission components of the robot. When the guidewire moves, the resistance will be transmitted to the force sensors.

Since the proximal force is a relatively complex resultant force, the robot's structure of the robot, the shape of the interventional device, and the different arrangement angles of the robot will all affect the resultant force. The reliability of the proximal force is poor, and it needs to be further processed by the algorithm to achieve the related function of force feedback.

\section{B. Master Controllers and Haptic Feedback}

The master controller is the most important part of the human-computer interaction. From the design perspective, it can be divided into master-slave isomorphic and master-slave heterogeneous. The former refers to the use of a completely different operation method from the interventional technique. Interventional doctors need more study and experimentation to adapt to its operation method, while the latter refers to using roughly the same operation method as the interventional technique. The interventional doctor can quickly become familiar with the operation of the robot and even reproduce the surgical habits or experience acquired in the manual interventional operation into the operation of the robot.

Many research groups have adopted commercial force feedback manipulators, such as Novint Falcon [36, 37], Phantom Omni [10, 27] and Geomagic Touch X [12, 13]. Commercial force feedback controllers have rich and stable force feedback functions, and it is simple and efficient to develop a haptic feedback system based on these controllers. But the disadvantage of using them for master controllers is also obvious; that is, they are general-purpose controllers, not specifically designed for interventional surgery. The experience accumulated by doctors in manual interventional surgery is difficult to apply to such controllers.

To get haptic feedback master controllers of the master-slave isomorphism, many research groups have developed their own master controllers. Tanimoto et al. of Nagoya University designed a rod-shaped controller in 2000, generating resistance to the doctor's hand through the connected motor [38, 39]. Since then, similar structures have been adopted by many research groups, such as Feng Zhenqiu et al. [40, 41] (Institute of Automation of the Chinese Academy of Sciences), Kundong Wang et al. [42, 43] (Shanghai Jiao Tong University), and Guo et al. $[11,24,44]$ (Kagawa University). 


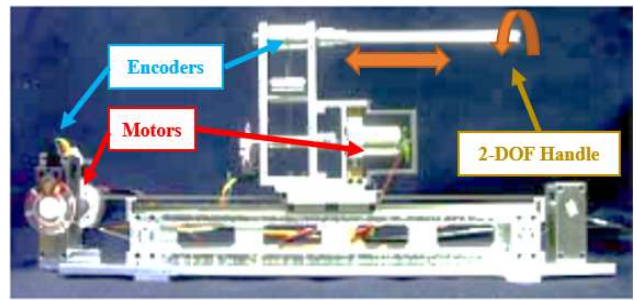

Fig. 11. Rod-shaped master controller designed by Tanimoto et al. The operator controls the rotation or delivery of the interventional instrument by rotating or pushing the handle. Both the rotation and linear movements of the handle will be recorded by encoders. At the same time, the internal motor can generate resistance to its handle.

Master-slave catheter controllers are another form of master controller design that puts a catheter in its master controller. The robot system will copy the actions from the master catheter to the slave catheter. This form of design makes the operation method of the robot completely consistent with the actual interventional surgery, which can greatly reduce the doctor's learning threshold and reproduce the interventional doctor's operation techniques and skills. Early master-slave catheter controllers only achieved master-catheter's motion detection, such as Tavallaei et al. [45] (The University of Western Ontario, London). Later, some groups have found ways to put resistance on the master-catheter. Guo et al. (Kagawa University) developed a haptic feedback system based on magnetorheological fluid [25, 46-48]. Payne et al. (Imperial College) added resistance to a master-catheter using a voice coil motor [29].

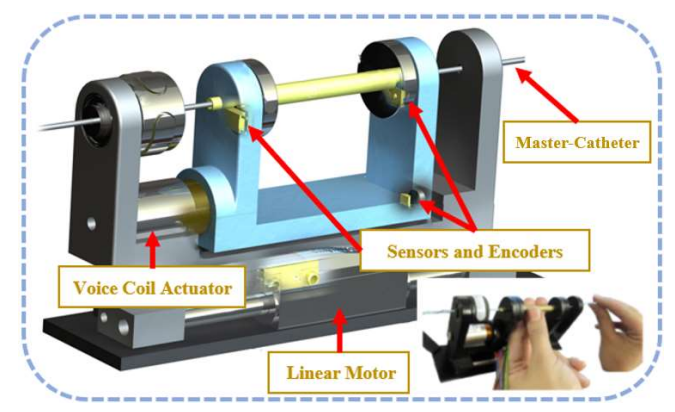

Fig. 12. This master-slave catheter controller with haptic feedback was designed by Payne et al. Its built-in sensors and encoders can detect the movement of mater-catheter and output resistance to the operator through voice coil actuator and linear motor.

\section{Safety Strategies}

In the interventional surgery robot system, the safest strategy is very important. During surgery, common dangerous situations include excessive contact force on the blood vessel wall, excessive deformation of surgical instruments, and doctor's incorrect operation. These dangerous situations will cause the patient to be injured, and the interventional surgical robot systems need to avoid such situations as much as possible.

Monitoring and controlling the force of interventional devices is a common force safety strategy. The simplest and most direct method is to measure the force through a force sensor and set a threshold for the feedback signal. Many research groups have adopted this approach, such as Guo Shuxiang et al. of Kagawa University [27], Cercenelli et al. of the University of Bologna [17] and Zhou et al. of Xiamen University [32]. The Corpath GRX system also has such a warning function. When excessive force is detected on the interventional device, a warning message will output on the user interface. At the same time, the system will prohibit the advancement of the device.

However, setting a threshold for the detected force is a crude approach. The force state of the interventional device is the result of a combination of multiple conditions. It is necessary to distinguish dangerous situations more accurately. Guo Shuxiang et al. of Beijing Institute of Technology proposed a neural network-based force safety method. The neural network will intelligently warn and take measures based on the resistance force and torque detected by the sensor to ensure the safety of surgery [49].

The abnormal deformation of surgical instruments is also very dangerous. Dagnino et al. of Imperial College London have introduced image recognition in their surgical robot system by identifying interventional images to track the position information of the guidewire and the blood vessel wall and make safety judgments, applying dynamic constraints [50].

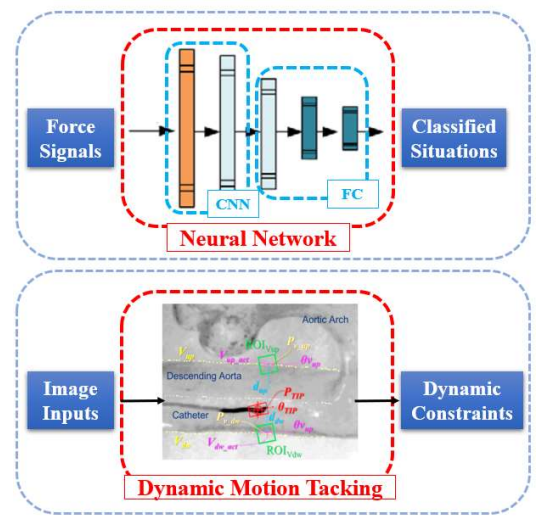

Fig. 13. In addition to the magnitude of the force, the current security situation is also related to the deep fuzzy information in the force signal. Using neural networks to learn these fuzzy information helps to judge the current security situation more accurately. By observing the position and posture of the guidewire and the state of the vascular environment through the image, the safety state can also be judged.

The doctor's incorrect operation can have a fatal impact on the patient. Hao Shen et al. of Shanghai Jiaotong University designed an "eccentric spring" algorithm to reduce the error signal input to the controller caused by trembling hands [51]. Yujia Xiang et al. used a Kalman filter in their algorithm to eliminate the tremor signal of the doctor's hand [52]. Guo Shuxiang et al. of Beijing Institute of Technology used a support vector machine (SVM) to identify the input operation signal to detect signals caused by trembling hands [53]. At the same time, they designed an algorithm applied to the master controller by magnetorheological fluid to resist hand tremors [47]. To prevent the misoperation from causing serious injury to the patient, they designed a braking device that can stop the movement of the surgical instrument in time when the safety system detects a misoperation [54]. 


\section{Control Methods}

To separate the operator from the radiation environment, the hardware structure of the interventional surgery robot system is often composed of an upper computer and a lower computer. The upper computer is placed in No radiation area and connected to the master controllers while the lower computer is placed in the radiation area and connected to the robot. In such an upper and lower system, the robot system adopts a masterslave method for control.

Open-loop speed control is a common control method. In this method, the input signal of the master controller is mapped to the speed space of the surgical robot and converted into the corresponding motor control signal. The mapping space is often divided into multiple sections to reduce excessive speed changes and jitter caused by trembling hands or misoperations. Fig. 14 shows the control mapping curve of Hao Shen et al. (Shanghai Jiaotong University) and Zhou et al. (Xiamen University). Such open-loop speed control is relatively simple.

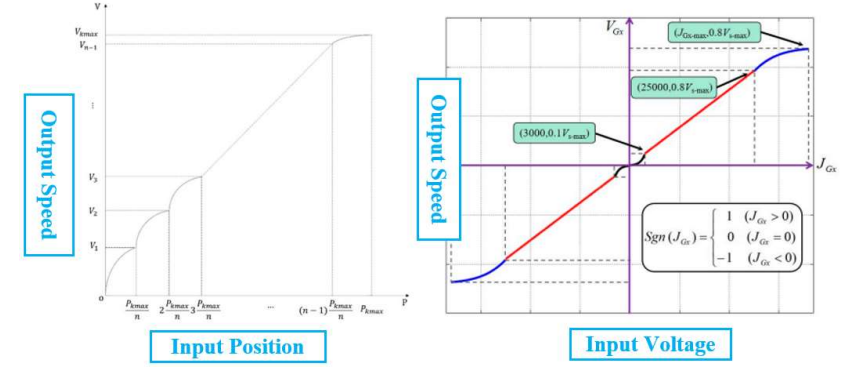

Fig. 14. (a) Control mapping curve of Hao Shen et al. They mapped the input position of an Omega 3 controller handle to the speed of surgery robot [51, 55]. (b) The mapping curve of Zhou et al. They mapped the input voltage from a commercial joystick to the speed of catheter and guidewire [32].

If the surgical robot system is equipped with position or angle sensors, more precise and stable closed-loop control can be achieved. Closed-loop control is more used in position or angle control; that is, the input signal of the master controller is mapped to the position space of the surgical robot and converted into the corresponding motor control signal. At the same time, the control system compares the feedback position of the sensor to achieve accuracy. Commonly used position sensors include angle encoders, position sensors of linear platforms, and electromagnetic tracking sensors, such as NDI Aurora.

Suppose the motion of the control object of the surgical robot is complicated, such as an electrophysiological catheter that can be bent at the front end. In that case, the relationship between the input of the robot system actuator and the output position of the interventional instrument can be solved by building a kinematic model $[21,55,56]$. In addition to control methods based on classical control theory, the use of control methods in modern control theory can sometimes improve the control system's performance. For example, Xiaomei Wang et al. of the University of Hong Kong have used a control method based on optimal control in their interventional surgery robot system. Their experiments show that the method's performance based on the optimal control theory is better than the control method based on the kinematic model [57].

PID control is the most used feedback control method. It performs proportional, integral, and derivative of the difference between the target value and the measured actual position value and combines these control variables to achieve stable tracking of the controlled object. Many research groups use PID controllers for surgical robot control [29, 45]. Based on PID control, setting fuzzy rules to dynamically adjust the PID controller parameters can realize more sensitive and stable fuzzy adaptive PID control. Wang et al. of Yanshan University applied a fuzzy PID controller in their surgical robot system and the fuzzy PID controller of them shown better performance than the traditional PID controller $[58,59]$.

\section{FRONTIERS OF VASCULAR INTERVENTIONAL ROBOTS}

\section{A. Automation surgeries}

Traditional master-slave interventional surgery robots perform actions that generally depend on the operator input actions, so surgery result depends on the operator's performance. There are often mistakes in manual operation. The mistakes potentially reduce the efficiency. Human reaction abilities, accuracy, and flexibility are far weaker than those of robot systems. In the future, autonomous surgery might even replace doctors to save the hospital's human resources. The goal of autonomous control technology is to enable robot systems to complete part of the surgery operation process or even completely take over the operation.

To achieve autonomous delivery, a common method is to specify a delivery rule to deliver based on the state of the interventional device. Jayender et al. (Weston University) proposed an autonomous method by machine vision $[56,60]$. Through image recognition of the path of the in vitro model, the robot can autonomously deliver the catheter to the target location. Corindus has introduced an FDA-approved guidewire autonomous feature known as "Rotate-on-Retract". This function is to rotate the guidewire whenever it is retracted by the operator, changing the guidewire tip's orientation in preparation for the next advancement [61].

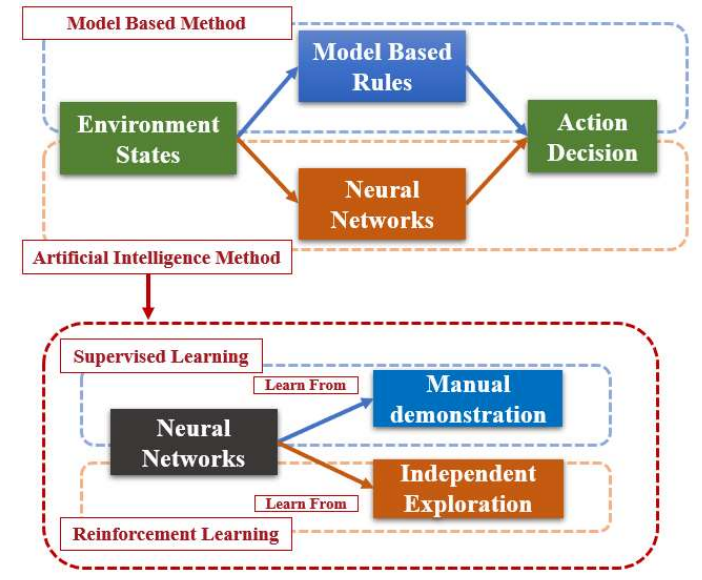

Fig. 15. The environment of intravascular surgery is quite complex, and modelbased methods may be difficult to make complex and ambiguous decisions. AIbased methods can learn fuzzy environmental regulations through supervised learning or reinforcement learning and make more complex decisions.

However, the environment of interventional surgery is very complicated, rules set by people can handle very limited situations. Moreover, many operate experience of interventional surgery is very vague and difficult to describe with specific rules. Using artificial intelligence to learn how to 
operate interventional devices from the experience of experts or self-exploration is an emerging method. Guo et al. (Beijing Institute of Technology) proposed an intelligent autonomous interventional surgery agent by using neural networks to learn from the operate records of multiple human experts.

In addition to learning from human demonstrations, agent can also learn from self-exploration, which called reinforcement learning. Through reinforcement learning, it is even possible to obtain an agent whose ability far exceeds that of human beings. Chi, Wenqiang et al. (Imperial College) applied reinforcement learning methods to their artificial intelligence agent. They used the most advanced algorithm in the field of reinforcement learning named proximal policy optimization, and their agent performs better than human expert in vitro models [62]. The application of reinforcement learning in various fields has shown outstanding performance, autonomous interventional surgery agents based on reinforcement learning are very promising.

\section{B. Long Geographic Distances Robotic Telestenting}

The Long Geographic Distances Robotic Telestenting of interventional surgical robots has received more and more attention in recent years. Using remote communication to allow doctors to perform long geographic distances remote operations on patients in another place can alleviate the uneven geographical distribution of interventional doctors, optimize human resources, and popularize interventional operations in various regions. Today's Internet technology is very mature, especially the emerging $5 \mathrm{G}$ network technology, which can fully meet the needs of low latency and large data throughput required by Internet surgery.

Guo Shuxiang et al. built a cloud server platform for their surgical robot platform, verifying that the cloud server can fully meet the needs of remote surgery [63]. Based on the Corpath GRX surgical robot system, Madder et al. performed the first remote model intervention experiment and animal in vivo intervention experiment in 2018, which confirmed the safety and feasibility of remote intervention surgery [64]. In 2020, they used wired networks and $5 \mathrm{G}$ wireless networks to perform remote transcontinental intervention experiments and succeeded [65].

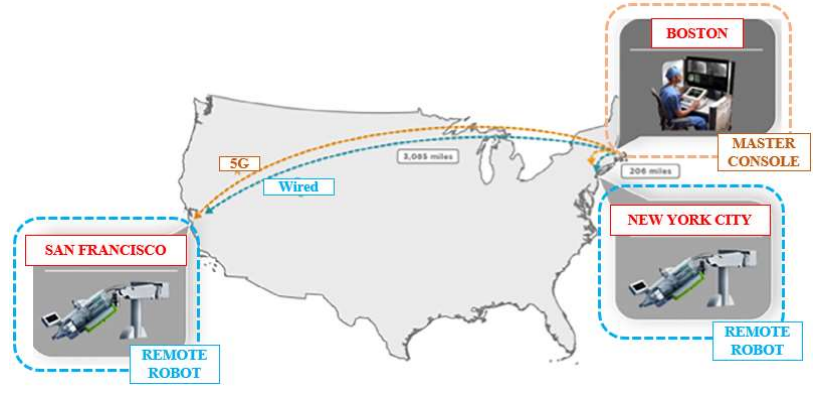

Fig. 16. Madder et al.'s experiments in 2018. By trying wired connection and $5 \mathrm{G}$ connection, they successfully completed the remote surgery experiment in two places separated by more than 3000 miles.

\section{MRI Compatible}

The fusion of interventional surgical robots and magnetic resonance imaging (MRI) navigation is also a research direction of several institutions. Since MRI does not have fluorescent radiation on the human body, children, pregnant women, and other people who are sensitive to radiation are more suitable for interventional surgery under MRI. Although the MRI environment will not pose any threat to the health of interventional surgeons, the application of surgical robots can still reduce the labor burden of doctors and improve the performance of surgery.

However, ferromagnetic and conductive materials cannot be compatible with the MRI environment, so designing such robot is challenging. A feasible solution is to place the power source outside the MRI operating room and transmitting the power to the operating room through MRI-compatible mediums. For example, Ka-Wai Kwok et al. (University of Hong Kong) designed a hydraulic Interventional electrophysiological robot [57, 66, 67], and Abdelaziz et al. (Imperial College, London) designed a pneumatic interventional robot [68]. The bodies and internal transmission parts of these robots are made of plastic or other materials compatible with the MRI environment.

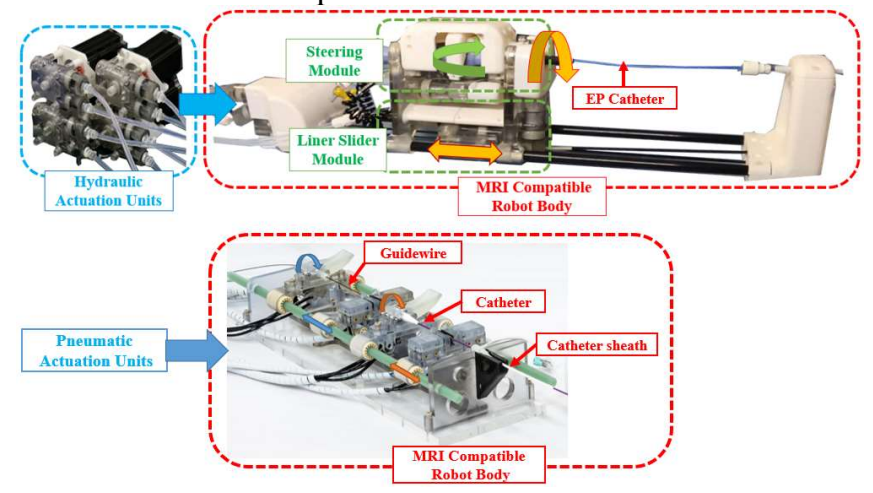

Fig. 17. MRI-compatible interventional robot by Kwok et al. used for electrophysiological treatment (a) and Abdelaziz et al. used for general vasculature interventional treatment (b). This type of design is quite challenging in separating the actuator (with ferromagnetic and conductive materials) from the surgical robot and transferring the power through MRI-compatible mediums.

\section{PROSPECTS AND CONCLUSIONS}

At present, the vascular interventional surgical robot system has mostly fulfilled the demand for remote manipulation of surgical instruments and is moving in the direction of stronger compatibility with interventional instruments and with more surgical functions. In scientific research, the research on the mechanical structure and control methods of robots has gradually matured. Still, more in-depth development is needed in terms of haptic feedback, safety strategies, and autonomous delivery strategies. With the continuous research and development of the vascular interventional surgical robot system, the future surgical robot system is expected to be more perfect in the following aspects:

1. A more compatible robot system for vascular interventional surgery. There are many surgical options for vascular intervention, and the surgical instruments used are also different. The future vascular interventional surgical robot system will be compatible with more surgical instruments, with richer functions and corresponding modules, enabling more surgical procedures, and further reducing the manual operations required by doctors.

2. Richer sensor information. It is very important to add more relevant sensors through the design to monitor the surgical 
status in greater detail. The contact force between the surgical instrument and the blood vessel wall, the specific position and shape of the catheter and the guidewire, the shape and pressure of the blood vessel lumen, and other complex information have important guiding significance for the surgical robot system.

3. More powerful master controllers. The hand perception of interventional doctors plays an important role in guiding their operations, and the rich surgical experience of interventional doctors is also extremely important. Therefore, the more precise and reliable haptic feedback and the design of the master manipulator that is more in line with the operating habits of interventional doctors can make it easier for interventional doctors to use the robot system and improve operating efficiency.

4. Long geographic distances robotic telestenting. With the development of communication technology and the popularization of $5 \mathrm{G}$ Internet technology, remote surgery technology may be an important feature of surgical robot systems in the future. Remote surgery can alleviate the problem of uneven distribution of human resources for interventional doctors. Experienced interventional doctors can remotely perform interventional operations on patients from far away.

5. A training and teaching system for vascular interventional surgery robots combined with virtual reality technology. Through virtual reality technology, blood vessel models, interventional instruments, and robot mechanisms can be established in a virtual environment. Students can experience the simulated interventional surgery process and practice the operating methods of surgical robots through only one manipulator.

6. Extensive application of artificial intelligence. With the accelerating development in artificial intelligence in recent years, more and more artificial intelligence methods will be applied to interventional surgical robot systems, such as medical image recognition, robot sensor information analysis and safety warning, and autonomous intelligent surgery.

Vascular interventional surgery robotics has huge potential. In the future, it will inevitably replace many interventional doctors in the operating room, reduce the workload of interventional doctors, and complete the surgical goals more efficiently and safely. Table I lists researches of vascular interventional surgical robots.

TABLE I

RESEARCHES OF INTERVENTIONAL SURGICAL ROBOTS

\begin{tabular}{|c|c|c|c|c|}
\hline Research institute & Field & $\begin{array}{l}\text { Haptics } \\
\text { feedback }\end{array}$ & $\begin{array}{c}\text { Force } \\
\text { sensing }\end{array}$ & $\begin{array}{c}\text { Autonomous } \\
\text { delivery }\end{array}$ \\
\hline $\begin{array}{l}\text { University of Bologna } \\
{[17-19]}\end{array}$ & EP & & Distal & $\checkmark$ \\
\hline $\begin{array}{l}\text { University of Hong } \\
\text { Kong } \\
{[57,66,67]}\end{array}$ & EP & & & \\
\hline $\begin{array}{l}\text { Korea University } \\
\text { [69] }\end{array}$ & EP & & & \\
\hline $\begin{array}{l}\text { Harbin Institute of } \\
\text { Technology } \\
{[36,70]}\end{array}$ & EP & $\checkmark$ & Proximal & \\
\hline $\begin{array}{l}\text { Weston University } \\
{[45,56,60,71-76]}\end{array}$ & $\begin{array}{l}\text { EP, } \\
\text { GV }\end{array}$ & & & $\checkmark$ \\
\hline $\begin{array}{l}\text { University of Waterloo } \\
\text { [21] }\end{array}$ & EP & & & $\checkmark$ \\
\hline $\begin{array}{l}\text { Beijing University of } \\
\text { Aeronautics and } \\
\text { Astronautics } \\
{[30,77-81]}\end{array}$ & GV & $\checkmark$ & Distal & \\
\hline
\end{tabular}

Yanshan University

$[58,59,82-85]$

Tianjin University

[86]

Shanghai Jiao Tong

University

$[42,43,51,52,55,87-$

89]

Shanghai University

[90]

Xiamen University

[32]

Saitama Institute of

Technology

[91]

University of Illinois

Urbana Champaign

$[34,35,92]$

Shenzhen Institutes of

Advanced Technology,

Chinese Academy of

Sciences

$[28,93,94]$

Institute

Automation, Chinese

$[40,41,95,96]$

Hanyang University

[97-101]

Imperial

London

$[29,50,62,68,102-$

$108]$

Beijing Institute of

Technology

$[12,13,25,44,49,53$,

$54,63,109-111]$

Nagoya University

$[38,39,112-114]$

State University of

New York

[115]

Katholieke

Universiteit Leuven

[116]

Kagawa University

$[10,11,26,27,46-48]$

Harbin Engineering

University

[117]
Academy of Sciences

\begin{tabular}{|c|c|c|}
\hline GV & $\checkmark$ & Proximal \\
\hline GV & & Distal \\
\hline GV & $\checkmark$ & Proximal \\
\hline GV & $\checkmark$ & Proximal \\
\hline GV & & Proximal \\
\hline GV & $\checkmark$ & Proximal \\
\hline GV & $\checkmark$ & Proximal \\
\hline GV & $\checkmark$ & $\begin{array}{c}\text { Proximal, } \\
\text { Distal }\end{array}$ \\
\hline GV & $\checkmark$ & \\
\hline GV & $\checkmark$ & Proximal \\
\hline GV & $\checkmark$ & Distal \\
\hline GV & $\checkmark$ & $\begin{array}{c}\text { Proximal, } \\
\text { Distal }\end{array}$ \\
\hline GV & $\checkmark$ & $\begin{array}{c}\text { Proximal, } \\
\text { Distal }\end{array}$ \\
\hline GV & $\checkmark$ & Proximal \\
\hline TAVI & & \\
\hline GV & $\checkmark$ & $\begin{array}{c}\text { Proximal, } \\
\text { Distal }\end{array}$ \\
\hline GV & & \\
\hline
\end{tabular}

\section{REFERENCES}

[1] Miller, Donald L. "Overview of contemporary interventional fluoroscopy procedures." Health physics 95.5 (2008): 638-644.

[2] Miller, Donald L., et al. "Occupational radiation protection in interventional radiology: a joint guideline of the Cardiovascular and Interventional Radiology Society of Europe and the Society of Interventional Radiology." Cardiovascular and interventional radiology 33.2 (2010): 230-239.

[3] Ross, Allan M., et al. "Prevalence of spinal disc disease among interventional cardiologists." American Journal of Cardiology 79.1 (1997): 68-70.

[4] Rafii-Tari, Hedyeh, Christopher J. Payne, and Guang-Zhong Yang. "Current and emerging robot-assisted endovascular catheterization technologies: a review." Annals of biomedical engineering 42.4 (2014): 697-715.

[5] Abdullah, Obai, et al. "Percutaneous angioplasty versus atherectomy for treatment of symptomatic infra-popliteal arterial disease." Cardiovascular Revascularization Medicine 19.4 (2018): 423-428.

[6] Mukai, Moe, et al. "Cryothermal atrial linear ablation in patients with atrial fibrillation: An insight from the comparison with radiofrequency atrial linear ablation." Journal of cardiovascular electrophysiology 31.5 (2020): $1075-1082$. 
[7] Matsuoka, Toshiyuki, and Tomohisa Okuma. "CT-guided radiofrequency ablation for lung cancer." International journal of clinical oncology 12.2 (2007): 71-78

[8] Won, Jong Yun, Byung-Ju Yi, and Hyo-Jeong Cha. "Review of New Master/Slave Catheter Driving Vascular Intervention Robot System: Intervention Radiologist's Perspective." Hanyang Medical Reviews 36.4 (2016): 225-229.

[9] Madder, Ryan D., et al. "Impact of robotics and a suspended lead suit on physician radiation exposure during percutaneous coronary intervention." Cardiovascular Revascularization Medicine 18.3 (2017): 190-196.

[10] Guo, Shuxiang, et al. "A novel active catheter system for ileus treatment." 2008 IEEE International Conference on Automation and Logistics. IEEE, 2008.

[11] Guo, Jian, et al. "A novel robotic catheter system with force and visual feedback for vascular interventional surgery." International Journal of Mechatronics and Automation 2.1 (2012): 15-24.

[12] Bao, Xianqiang, et al. "Operation evaluation in-human of a novel remotecontrolled vascular interventional robot." Biomedical microdevices 20.2 (2018): 1-13.

[13] Bao, Xianqiang, et al. "A cooperation of catheters and guidewires-based novel remote-controlled vascular interventional robot." Biomedical microdevices 20.1 (2018): 1-19.

[14] "China's first interventional robot-assisted DSA operation completed in Tiantan Hospital." Care Health $\{3\} .01(2020): 96$. doi:CNKI:SUN:JKZJ.0.2020-01-038.

[15] Beyar, Rafael, et al. "Remote-control percutaneous coronary interventions: concept, validation, and first-in-humans pilot clinical trial." Journal of the American College of Cardiology 47.2 (2006): 296-300.

[16] Robocath "The Medical Robot R-One [Online] Available: https://www.robocath.com/product/.

[17] Cercenelli, Laura, Barbara Bortolani, and Emanuela Marcelli. "CathROB: A highly compact and versatile remote catheter navigation system." Applied Bionics and Biomechanics 2017 (2017).

[18] Marcelli, E., L. Cercenelli, and G. Plicchi. "A novel telerobotic system to remotely navigate standard electrophysiology catheters." 2008 Computers in Cardiology. IEEE, 2008.

[19] Cercenelli, Laura, Emanuela Marcelli, and Gianni Plicchi. "Initial experience with a telerobotic system to remotely navigate and automatically reposition standard steerable EP catheters." Asaio Journal 53.5 (2007): 523-529.

[20] Park, Jun Woo, et al. "Development of a force-reflecting robotic platform for cardiac catheter navigation." Artificial organs 34.11 (2010): 1034-1039.

[21] Ganji, Yusof, Farrokh Janabi-Sharifi, and Asim N. Cheema. "Robotassisted catheter manipulation for intracardiac navigation." International journal of computer assisted radiology and surgery 4.4 (2009): 307-315.

[22] Heunis, Christoff, Jakub Sikorski, and Sarthak Misra. "Flexible instruments for endovascular interventions: Improved magnetic steering, actuation, and image-guided surgical instruments." IEEE robotics \& automation magazine 25.3 (2018): 71-82.

[23] Stereotaxis "The Genesis RMN System." Accessed on: Jun. 30, 2021, [Online] Available: https://www.stereotaxis.com/products/.

[24] Guo, Jian, Shuxiang Guo, and Yang Yu. "Design and characteristics evaluation of a novel teleoperated robotic catheterization system with force feedback for vascular interventional surgery." Biomedical microdevices 18.5 (2016): 1-16

[25] Guo, Shuxiang, et al. "A novel robot-assisted endovascular catheterization system with haptic force feedback." IEEE Transactions on Robotics 35.3 (2019): 685-696

[26] Guo, Jian, et al. "A novel type of catheter sidewall tactile sensor array for vascular interventional surgery." 2013 ICME international conference on complex medical engineering. IEEE, 2013.

[27] Guo, Jian, et al. "A force display method for a novel catheter operating system." The 2010 IEEE International Conference on Information and Automation. IEEE, 2010.

[28] Omisore, Olatunji Mumini, et al. "Towards characterization and adaptive compensation of backlash in a novel robotic catheter system for cardiovascular interventions." IEEE transactions on biomedical circuits and systems 12.4 (2018): 824-838.

[29] Payne, Christopher J., Hedyeh Rafii-Tari, and Guang-Zhong Yang. "A force feedback system for endovascular catheterisation." 2012 IEEE/RSJ International Conference on Intelligent Robots and Systems. IEEE, 2012.

[30] Zhao, Depeng, and Da Liu. "Fuzzy fusion of forces feedback in vascular interventional surgery robot system." Robot 35.1 (2013): 60-66.
[31] [Online] Available: http://www.hansenmedical.com/us/en/cardiacarrhythmia/artisan-extendcatheter/product-overview

[32] Zhou, Junqiang, et al. "A remote-controlled robotic system with safety protection strategy based on force-sensing and bending feedback for transcatheter arterial chemoembolization." Micromachines 11.9 (2020): 805.

[33] Cha, Hyo-Jeong, Byung-Ju Yi, and Jong Yun Won. "An assembly-type master-slave catheter and guidewire driving system for vascular intervention." Proceedings of the Institution of Mechanical Engineers, Part H: Journal of Engineering in Medicine 231.1 (2017): 69-79.

[34] Sankaran, Naveen Kumar, Pramod Chembrammel, and Thenkurussi Kesavadas. "Force calibration for an endovascular robotic system with proximal force measurement." The International Journal of Medical Robotics and Computer Assisted Surgery 16.2 (2020): e2045.

[35] Sankaran, Naveen Kumar, et al. "Design and development of surgeon augmented endovascular robotic system." IEEE Transactions on Biomedical Engineering 65.11 (2018): 2483-2493.

[36] Fu, Yili, et al. "Development of a novel robotic catheter system for endovascular minimally invasive surgery." The 2011 IEEE/ICME International Conference on Complex Medical Engineering. IEEE, 2011.

[37] Fu, Yili, et al. "The master-slave catheterisation system for positioning the steerable catheter." International Journal of Mechatronics and Automation 1.3-4 (2011): 143-152.

[38] Arai, Fumihito, et al. "New catheter driving method using linear stepping mechanism for intravascular neurosurgery." Proceedings 2002 IEEE International Conference on Robotics and Automation (Cat. No. 02CH37292). Vol. 3. IEEE, 2002.

[39] Tanimoto, Mitsutaka, et al. "Telesurgery system for intravascular neurosurgery." International Conference on Medical Image Computing and Computer-Assisted Intervention. Springer, Berlin, Heidelberg, 2000.

[40] Feng, Zhen-Qiu, et al. "Design and evaluation of a bio-inspired robotic hand for percutaneous coronary intervention." 2015 IEEE International Conference on Robotics and Automation (ICRA). IEEE, 2015.

[41] Bian, Gui-Bin, et al. "An enhanced dual-finger robotic Hand for Catheter manipulating in vascular intervention: A preliminary study." 2013 IEEE International Conference on Information and Automation (ICIA). IEEE, 2013.

[42] Wang, Kundong, et al. "Force feedback controls of multi-gripper robotic endovascular intervention: design, prototype, and experiments." International Journal of Computer Assisted Radiology and Surgery 16.1 (2021): 179-192.

[43] Wang, Kundong, et al. "A novel SEA-based haptic force feedback master hand controller for robotic endovascular intervention system." The International Journal of Medical Robotics and Computer Assisted Surgery 16.5 (2020): 1-10.

[44] Xiao, Nan, Ping Guo, and Shuxiang Guo. "Push force feedback for a kind of robotic catheter navigation system." 2015 IEEE International Conference on Information and Automation. IEEE, 2015.

[45] Thakur, Yogesh, et al. "Design and performance evaluation of a remote catheter navigation system." IEEE Transactions on biomedical engineering 56.7 (2009): 1901-1908.

[46] Shi, Peng, et al. "A Two-channel Haptic Force Interface for Endovascular Robotic Systems." 2020 IEEE International Conference on Mechatronics and Automation (ICMA). IEEE, 2020.

[47] Zheng, Lingling, and Shuxiang Guo. "A magnetorheological fluid-based tremor reduction method for robot-assisted catheter operating system." International Journal of Mechatronics and Automation 8.2 (2021): 72-79.

[48] Yin, Xuanchun, et al. "Safety operation consciousness realization of a MR fluids-based novel haptic interface for teleoperated catheter minimally invasive neurosurgery." IEEE/ASME Transactions on Mechatronics 21.2 (2015): 1043-1054

[49] Wang, Yuxin, et al. "A CNNs-based of force and torque identification model for vascular interventional surgery robot." 2019 IEEE International Conference on Mechatronics and Automation (ICMA). IEEE, 2019.

[50] Dagnino, Giulio, et al. "Haptic feedback and dynamic active constraints for robot-assisted endovascular catheterization." 2018 IEEE/RSJ International Conference on Intelligent Robots and Systems (IROS). IEEE, 2018.

[51] Shen, Hao, et al. "A novel remote - controlled robotic system for cerebrovascular intervention." The International Journal of Medical Robotics and Computer Assisted Surgery 14.6 (2018): e1943.

[52] Xiang, Yujia, et al. "Master-Slave Guidewire and Catheter Robotic System for Cardiovascular Intervention." 2019 28th IEEE International 
Conference on Robot and Human Interactive Communication (RO-MAN). IEEE, 2019.

[53] Guo, Shuxiang, et al. "Recognition and Filtering of Tremor Signals for Vascular Interventional Surgical Robot." 2020 IEEE International Conference on Mechatronics and Automation (ICMA). IEEE, 2020.

[54] Yang, Cheng, and Shuxiang Guo. "A Novel Misoperation Preventing Device for the Interventional Surgical Robot." 2020 IEEE International Conference on Mechatronics and Automation (ICMA). IEEE, 2020.

[55] Shen, Hao, et al. "A novel robotic system for vascular intervention: principles, performances, and applications." International journal of computer assisted radiology and surgery 14.4 (2019): 671-683.

[56] Jayender, Jagadeesan, Rajnikant V. Patel, and Suwas Nikumb. "Robotassisted active catheter insertion: Algorithms and experiments." The International Journal of Robotics Research 28.9 (2009): 1101-1117.

[57] Wang, Xiaomei, et al. "Experimental validation of robot-assisted cardiovascular catheterization: model-based versus model-free control." International journal of computer assisted radiology and surgery 13.6 (2018): 797-804.

[58] Yu, Haoyang, et al. "A Novel Vascular Intervention Surgical Robot Based on Force Feedback and Flexible Clamping." Applied Sciences 11.2 (2021): 611.

[59] Wang, Hongbo, et al. "Fuzzy controller design of the wire feeder of invasive vascular interventional surgery robot." 2015 International Conference on Advanced Mechatronic Systems (ICAMechS). IEEE, 2015.

[60] Jayender, Jagadeesan, Mahdi Azizian, and Rajni V. Patel. "Autonomous image-guided robot-assisted active catheter insertion." IEEE Transactions on Robotics 24.4 (2008): 858-871.

[61] Al Nooryani, Arif, and Wael Aboushokka. "Rotate-on-retract procedural automation for robotic-assisted percutaneous coronary intervention: first clinical experience." Case reports in cardiology 2018 (2018).

[62] Chi, Wenqiang, et al. "Collaborative robot-assisted endovascular catheterization with generative adversarial imitation learning." 2020 IEEE International Conference on Robotics and Automation (ICRA). IEEE, 2020.

[63] Guo, Yangming, Shuxiang Guo, and Cheng Yang. "Feasibility Study on Cloud Communication Operation for an Interventional Surgery Robot." 2020 IEEE International Conference on Mechatronics and Automation (ICMA). IEEE, 2020.

[64] Madder, Ryan D., et al. "Feasibility of robotic telestenting over long geographic distances: a pre-clinical ex vivo and in vivo study." EuroIntervention 15.6 (2019): e510-e512.

[65] Madder, Ryan D., et al. "Robotic telestenting performance in transcontinental and regional pre-clinical models." Catheterization and Cardiovascular Interventions 97.3 (2021): E327-E332.

[66] Dong, Ziyang, et al. "High-performance continuous hydraulic motor for MR safe robotic teleoperation." IEEE Robotics and Automation Letters 4.2 (2019): 1964-1971.

[67] Lee, Kit-Hang, et al. "MR safe robotic manipulator for MRI-guided intracardiac catheterization." IEEE/ASME Transactions on Mechatronics 23.2 (2018): 586-595

[68] Abdelaziz, Mohamed EMK, et al. "Toward a versatile robotic platform for fluoroscopy and mri-guided endovascular interventions: A pre-clinical study." 2019 IEEE/RSJ International Conference on Intelligent Robots and Systems (IROS). IEEE, 2019.

[69] Park, Jun Woo, et al. "Development of a force-reflecting robotic platform for cardiac catheter navigation." Artificial organs 34.11 (2010): 1034-1039.

[70] Fu, Yili, et al. "The master-slave catheterisation system for positioning the steerable catheter." International Journal of Mechatronics and Automation 1.3-4 (2011): 143-152.

[71] Tavallaei, Mohammad Ali, et al. "Design, development and evaluation of a compact telerobotic catheter navigation system." The International Journal of Medical Robotics and Computer Assisted Surgery 12.3 (2016): $442-452$

[72] Tavallaei, Mohammad Ali, et al. "A magnetic-resonance-imagingcompatible remote catheter navigation system." IEEE Transactions on Biomedical Engineering 60.4 (2012): 899-905.

[73] Thakur, Yogesh, et al. "Right-side RF ablation using remote catheter navigation: experimental results in vivo." Journal of cardiovascular electrophysiology 23.1 (2012): 81-87.

[74] Thakur, Yogesh, David W. Holdsworth, and Maria Drangova. "Characterization of catheter dynamics during percutaneous transluminal catheter procedures." IEEE Transactions on Biomedical Engineering 56.8 (2009): 2140-2143.

[75] Thakur, Yogesh, et al. "A device for real-time measurement of cathetermotion and input to a catheter navigation system." Medical Imaging 2007:
Visualization and Image-Guided Procedures. Vol. 6509. International Society for Optics and Photonics, 2007.

[76] Jayender, Jagadeesan, Rajnikant V. Patel, and Suwas Nikumb. "Robotassisted catheter insertion using hybrid impedance control." Proceedings 2006 IEEE International Conference on Robotics and Automation, 2006. ICRA 2006.. IEEE, 2006.

[77] Meng, Cai, et al. "A remote - controlled vascular interventional robot: system structure and image guidance." The international journal of medical robotics and computer assisted surgery 9.2 (2013): 230-239.

[78] Lu, Wang-sheng, et al. "Experimental study of remote angiography using vascular interventional robot." 2013 ICME International Conference on Complex Medical Engineering. IEEE, 2013.

[79] Lu, Wang-sheng, et al. "Application study of medical robots in vascular intervention." The International Journal of Medical Robotics and Computer Assisted Surgery 7.3 (2011): 361-366.

[80] Wang, Tianmiao, Dapeng Zhang, and Liu Da. "Remote - controlled vascular interventional surgery robot." The International Journal of Medical Robotics and Computer Assisted Surgery 6.2 (2010): 194-201.

[81] Guo, Jian, Yue Sun, and Shuxiang Guo. "A Novel Trajectory Predicting Method of Catheter for the Vascular Interventional Surgical Robot." 2020 IEEE International Conference on Mechatronics and Automation (ICMA). IEEE, 2020.

[82] Wang, Hongbo, et al. "Catheter intervention manipulation system of minimally invasive robotic surgery." The 2011 International Conference on Advanced Mechatronic Systems. IEEE, 2011.

[83] Yang, Xue, et al. "Minimally invasive vascular interventional surgical robot system." 2011 IEEE International Conference on Mechatronics and Automation. IEEE, 2011.

[84] Wang, Hongbo, et al. "Design of propulsion mechanism for vascular interventional operation robot." 2018 International Conference on Advanced Mechatronic Systems (ICAMechS). IEEE, 2018.

[85] Liu, Haiyang, et al. "Mechanism design of the minimally invasive vascular interventional surgery robot system." 2017 IEEE International Conference on Cybernetics and Intelligent Systems (CIS) and IEEE Conference on Robotics, Automation and Mechatronics (RAM). IEEE, 2017.

[86] He, Chengbin, Shuxin Wang, and Siyang Zuo. "A linear stepping endovascular intervention robot with variable stiffness and force sensing." International journal of computer assisted radiology and surgery 13.5 (2018): 671-682.

[87] Lu, Qingsheng, et al. "A Novel Universal Endovascular Robot for Peripheral Arterial Stent-Assisted Angioplasty: Initial Experimental Results." Vascular and Endovascular Surgery 54.7 (2020): 598-604.

[88] Shen, Jie, et al. "Design and experiment of guide wire tele-manipulation system based on laser mouse sensor." Zhongguo yi Liao qi xie za zhi= Chinese Journal of Medical Instrumentation 36.1 (2012): 32-35.

[89] Wang, Kundong, et al. "Endovascular intervention robot with multimanipulators for surgical procedures: Dexterity, adaptability, and practicability." Robotics and Computer-Integrated Manufacturing 56 (2019): 75-84.

[90] Li, Long, et al. "Design and Performance Evaluation of a Novel Vascular Interventional Surgery Robot." 2018 IEEE International Conference on Robotics and Biomimetics (ROBIO). IEEE, 2018.

[91] Zakaria, Noor Ayuni Che, et al. "Development of foolproof catheter guide system based on mechatronic design." Production Engineering 7.1 (2013): 81-90.

[92] Paranjape, Makarand R., ed. New Perspectives in Indian Science and Civilization. Taylor \& Francis, 2019.

[93] OMISORE, Olatunji Mumini, et al. "A teleoperated robotic catheter system with motion and force feedback for vascular surgery." 2018 18th International Conference on Control, Automation and Systems (ICCAS). IEEE, 2018.

[94] Mi, Jin-peng, et al. "A vascular intervention assisted robot based on masterslave control mode." 2014 IEEE International Conference on Mechatronics and Automation. IEEE, 2014.

[95] Ji, Cheng, Zeng-Guang Hou, and Xiao-Liang Xie. "Guidewire navigation and delivery system for robot-assisted cardiology interventions." IEEE 10th International Conference on Cognitive Informatics and Cognitive Computing (ICCI-CC'11). IEEE, 2011.

[96] Huang, Rui-Jian, et al. "Path planning for surgery robot with bidirectional continuous tree search and neural network." 2019 IEEE/RSJ International Conference on Intelligent Robots and Systems (IROS). IEEE, 2019.

[97] Lee, Wonseo, et al. "Steering, Tunneling, and Stent Delivery of a Multifunctional Magnetic Catheter Robot to Treat Occlusive Vascular 
Disease." IEEE Transactions on Industrial Electronics 68.1 (2020): 391400.

[98] Woo, Jaehong, et al. "Advantage of steerable catheter and haptic feedback for a 5-DOF vascular intervention robot system." Applied Sciences 9.20 (2019): 4305.

[99] Cha, Hyo-Jeong, Byung-Ju Yi, and Jong Yun Won. "An assembly-type master-slave catheter and guidewire driving system for vascular intervention." Proceedings of the Institution of Mechanical Engineers, Part H: Journal of Engineering in Medicine 231.1 (2017): 69-79.

[100] Cha, H-J., et al. "A robotic system for percutaneous coronary intervention equipped with a steerable catheter and force feedback function." 2016 IEEE/RSJ International Conference on Intelligent Robots and Systems (IROS). IEEE, 2016.

[101]Cha, Hyo-Jeong, and Byung-Ju Yi. "Design concept of a micro robot delivery system." 2015 12th International Conference on Ubiquitous Robots and Ambient Intelligence (URAI). IEEE, 2015.

[102]Rafii-Tari, Hedyeh, et al. "Objective assessment of endovascular navigation skills with force sensing." Annals of biomedical engineering 45.5 (2017): 1315-1327.

[103]Rafii-Tari, Hedyeh, et al. "Towards automated surgical skill evaluation of endovascular catheterization tasks based on force and motion signatures." 2015 IEEE International Conference on Robotics and Automation (ICRA). IEEE, 2015.

[104]Rafii-Tari, Hedyeh, et al. "Learning-based modeling of endovascular navigation for collaborative robotic catheterization." International conference on medical image computing and computer-assisted intervention. Springer, Berlin, Heidelberg, 2013.

[105]Chi, Wenqiang, et al. "Learning-based endovascular navigation through the use of non-rigid registration for collaborative robotic catheterization." International journal of computer assisted radiology and surgery 13.6 (2018): 855-864.

[106]Chi, Wenqiang, et al. "Trajectory optimization of robot-assisted endovascular catheterization with reinforcement learning." 2018 IEEE/RSJ International Conference on Intelligent Robots and Systems (IROS). IEEE, 2018.

[107]Chi, Wenqiang, et al. "Trajectory optimization of robot-assisted endovascular catheterization with reinforcement learning." 2018 IEEE/RSJ International Conference on Intelligent Robots and Systems (IROS). IEEE, 2018.

[108]Molinero, M. Benavente, et al. "Haptic guidance for robot-assisted endovascular procedures: implementation and evaluation on surgical simulator." 2019 IEEE/RSJ International Conference on Intelligent Robots and Systems (IROS). IEEE, 2019.

[109]Zhou, Wei, Shuxiang Guo, and Zhengyang Chen. "A Novel Axial Force Feedback Device for the Master Manipulator of the Vascular Interventional Surgical Robot." 2020 IEEE International Conference on Mechatronics and Automation (ICMA). IEEE, 2020.

[110]Zhao, Yan, et al. "A CNN-based prototype method of unstructured surgical state perception and navigation for an endovascular surgery robot." Medical \& Biological Engineering \& Computing 57.9 (2019): 1875-1887.

[111]Zhao, Yan, et al. "A novel noncontact detection method of surgeon's operation for a master-slave endovascular surgery robot." Medical and Biological Engineering and Computing 58.4 (2020): 871-885.

[112] Tercero, Carlos, et al. "Autonomous catheter insertion system using magnetic motion capture sensor for endovascular surgery." The International Journal of Medical Robotics and Computer Assisted Surgery 3.1 (2007): 52-58.

[113]Tanimoto, Mitsutaka, et al. "Micro force sensor for intravascular neurosurgery and in vivo experiment." Proceedings MEMS 98. IEEE. Eleventh Annual International Workshop on Micro Electro Mechanical Systems. An Investigation of Micro Structures, Sensors, Actuators, Machines and Systems (Cat. No. 98CH36176. IEEE, 1998.

[114]Guo, Shuxiang, et al. "Micro catheter system with active guide wire." Proceedings of 1995 IEEE International Conference on Robotics and Automation. Vol. 1. IEEE, 1995.

[115] Srimathveeravalli, Govindarajan, Thenkurussi Kesavadas, and Xinyan Li. "Design and fabrication of a robotic mechanism for remote steering and positioning of interventional devices." The International Journal of Medical Robotics and Computer Assisted Surgery 6.2 (2010): 160-170.

[116]Rosa, Benoit, et al. "Intuitive teleoperation of active catheters for endovascular surgery." 2015 IEEE/RSJ International Conference on Intelligent Robots and Systems (IROS). IEEE, 2015.

[117]Feng, Weixing, et al. "Highly precise catheter driving mechanism for intravascular neurosurgery." 2006 International Conference on Mechatronics and Automation. IEEE, 2006.

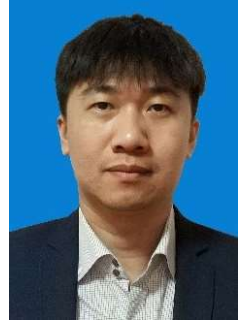

Yang Zhao received the Doctorate degree from the School of Mechancial Engineering, Jilin University, Changchun, China, in 2009.

He is currently an Associate Professor of Department of Mechanical \& Electrical Engineering, Xiamen University, Xiamen, $\mathrm{He}$ is specialized in the medical robotics of transcatheter arterial chemoembolization and medical sensors.

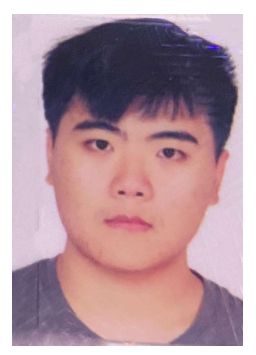

Ziyang Mei was born in 1997. He received the Baccalaureate degree from the Department of mechanical \& electrical engineering, Xiamen University Xiamen, China., in 2019.

$\mathrm{He}$ is currently a master student in the Department of mechanical \& electrical engineering, Xiamen University Xiamen, China. His research interests include Mechatronics design of medical robot system, as well as deep learning technology.

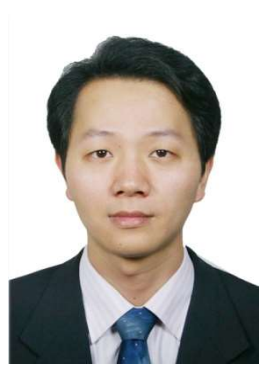

Dezhi Wu was born in 1977. He received the B.S. and M.S. degrees in mechanical and electronic engineering, Wuhan University of Technology, Wuhan, China., in 2003 and the Ph.D. degree in measuring and testing technologies and instruments from Xiamen University, Xiamen, China., in 2009.

He is currently a professor of Xiamen University, China. His research interests include soft robots, electronic skin, micro-nano fabrication technology and equipment, etc. He has presided over the National Natural Science Foundation of China. Shenzhen Science and Technology R\&D Fund Project and so on. Up to now, he has published more than 60 papers in internationally renowned journals such as Nanoscale. Organic Electronics, etc. and has about 20 authorized patents. 